\title{
Parafunctional Behaviors and Its Effect on Dental Bridges
}

\author{
Amal Alharby a, g, Hanan Alzayer ${ }^{b, g}$, Ahmed Almahlawic, Yazeed Alrashidid, Samaa Azharc, \\ Maan Sheikho ${ }^{\mathrm{d}}$, Anas Alandijanie, Amjad Aljohani ${ }^{\mathrm{f}}$, Manal Obied ${ }^{\mathrm{a}}$
}

\begin{abstract}
Parafunctional behaviors, especially bruxism, are not uncommon among patient visiting dentists' clinics daily and they constitute a major dental issue for almost all dentists. Many researchers have focused on the definition, pathophysiology, and treatment of these behaviors. These parafunctional behaviors have a considerable negative impact on teeth and dental prothesis. In this review, we focused on the impact of parafunctional behaviors on dental bridges. We summarized the definitions, epidemiology, pathophysiology, and consequences of parafunctional behaviors. In addition, we reviewed previous dental literature studies that demonstrated the effect of bruxism or other parafunctional behaviors on dental bridges and dental prothesis. In conclusion, parafunctional behaviors are common involuntary movements involving the masticatory system. They are more prevalent among children. These behaviors have deleterious effects on dental structures. Causes of parafunctional behaviors include anxiety, depression, smoking, caffeine intake, sleep disorders, or central neurotransmitter dysfunction. Bruxism and other similar masticatory system activity cause dental fracture, loss, and weardown of enamel or teeth. They can also affect different types of dental protheses both fixed and removable types. Parafunctional behaviors shorten the life expectancy of these protheses, and damage residual dentition and denture-bearing tissues.
\end{abstract}

Keywords: Parafunction; Parafunctional habits; Parafunctional behaviors; Dental; Bridge; Bruxism

\section{Introduction}

The masticatory system functions in one of two ways: a

Manuscript submitted December 8, 2017, accepted December 22, 2017

aDepartment of Dentistry, Riyadh Colleges of Dentistry and Pharmacy, Riyadh, Saudi Arabia

${ }^{b}$ Department of Dentistry, Primary Health Care, Qatif, Saudi Arabia

'Department of Dentistry, Ibn Sina National College, Jeddah, Saudi Arabia

${ }^{\mathrm{d}}$ Department of Dentistry, Prince Sattam Bin Abdulaziz University, Al-Kharj, Saudi Arabia

eDepartment of Dentistry, Umm Al-Qura University, Mecca, Saudi Arabia fDepartment of Dentistry, Buraydah Colleges, Buraydah, Saudi Arabia gCorresponding Author: Amal Alharby, Department of Dentistry, Riyadh Colleges of Dentistry and Pharmacy, Riyadh, Saudi Arabia. Email: aalharbiamal@gmail.com; Hanan Alzayer, Department of Dentistry, Primary Health Care, Qatif, Saudi Arabia. Email: noun_222@hotmail.com

doi: https://doi.org/10.14740/jocmr3304w functional and a parafunctional way. Functional activity includes meaningful work such as speaking, eating, or chewing, whereas parafunctional behaviors indicate abnormal hyperactive functions conducted by the masticatory structures, i.e. tongue, teeth, oral muscles, etc. [1]. Bruxism (teeth grinding), clenching, thump/digit suckling, lip or fingernail biting, and non-nutritive suckling exemplify parafunctional habits [2]. Functional activities are vital to smoothly perform essential functions of the oromandibular system without damaging it. On the other hand, parafunctional behaviors do not deliver a necessary function and they may lead to local tissue damage. The mechanism of parafunctional behaviors is different from functional activity [3].

\section{Types of Parafunctional Behaviors}

Given its close relation to dental field, bruxism is the parafunctional that has received special attention among researchers during the last three decades. Bruxism refers to teeth grinding or clenches that have deleterious effects on teeth or dental protheses. Next came non-nutritive suckling in which patients tend to suckle non-food items, particularly his thumb, any digit, or pacifiers in children. These behaviors disappear insidiously over time with progression of age. However, they may persist to the adult stage leading to significant damage of the masticatory and oromandibular structures. Non-nutritive suckling habit can easily be recognized through inspecting the teeth. Teeth of these individuals have pressure marks from the tearing mechanical force continuously applied to these teeth. Anterior open bite or posterior crossbite signs are also not uncommon. It is generally recommended to start active intervention to obligatorily cease these habits at the age of 3 years [4]. Other parafunctional behaviors include nail biting and lip or cheek biting [4].

\section{Prevalence of Parafunctional Behaviors}

Results from a research conducted on Saudi adolescents during the year 2006 depicted that lip and cheek biting was the most prevalent parafunctional behavior reported by $41 \%$ of participants. Nail biting was the second most common behavior having a figure of $29 \%$. Both bruxism and thumb suckling constituted only $15 \%$ of the cases [5].

Sleep bruxism occurs in about $7-8 \%$ of the general population. It is more prevalent in children occurring in up to $40 \%$ of children around age of 11 years, and it is estimated to be more 
prevalent among females [6]. A more detailed research about bruxism showed that $20 \%$ of adults had bruxism (teeth clenching) during awakening, only $8 \%$ of bruxers are aware of their night bruxism. Noisy bruxism was reported in $14-20 \%$ of participating children below the age of 11 years. Of the affected participants, $10-20 \%$ had signs of bruxism during conducting the research [7].

\section{Pathophysiology of Parafunctional Behavior}

In spite of continuous efforts to understand the nature and mechanisms of parafunctional behaviors, an exact pathophysiology remains elusive. Researches have proposed many theories for the development, or more accurately the persistence, of parafunctional behavior. The most common theory is the psychological hypothesis that the parafunctional activity represents a regression to or maintenance at an oral stage of development in which the mouth and face are used to vent the individual's stresses, frustrations, or anger [8]. Dopamine, serotonin, and noradrenaline are common neurotransmitters involved. Genetic causes were also studied [9]. Recent studies suggest that parafunctional behaviors develop due to central regulatory mechanisms and peripheral factors do not contribute to the pathophysiology [10].

\section{Bruxism Definitions}

Many definitions have been established for bruxism. According to the American Academy of Orofacial Pain, bruxism is a parafunctional activity such as grinding, clinching, gnashing or bracing of teeth which may occur either diurnal or nocturnal [11]. Diurnal and nocturnal bruxism are different entities, with different etiologies, pathophysiology, risk factors, and management plans [12]. American sleep disorders association adds to aforementioned definition the occurrence of one more feature such as tooth wear, tooth sounds, or discomfort of jaw muscles not explained by another medical illness [13]. The glossary of prosthodontic terms, on the other hand, defines bruxism as an oral habit in which an individual involuntarily and rhythmically grinds or clenches his or her teeth. Such habit might cause dental trauma, often referred to as "occlusal neurosis" [14]. When this grinding occurs during the waking stage, it is called "bruxomania" [15]. Clenching is not an equivalent to grinding. Clenching is a centric bruxism, whereas grinding is an eccentric bruxism. Clenching is a static process of forceful closure of mandible and maxilla, so that the opposing teeth meet at an eccentric position. On the other hand, grinding features a dynamic state during which the mandibular arch moves at different positions causing sliding of dentation over each other [15].

\section{Etiological and Risk Factors of Bruxism}

Many factors have also been hypothesized to be associated with bruxism including anatomical abnormalities, psycho- logical factors, sleep disorders, and medication side effects [6, 8-10]. Abnormal anatomy of the oromandibular and stomatognathic system can cause bruxism. The most common abnormality encountered among patients with bruxism is malalignment between upper and lower mandibles causing a bad occlusion. Psychological factors seem to play a key role in development of bruxism. Studies indicated that bruxer individuals had higher rates of anxiety and depression than their counterparts who do not have bruxism [10]. Patients with nocturnal bruxism often have other sleep disorders especially sleep apnea and snoring [16]. Other factors proposed include trauma, genetics, smoking, alcohol, caffeine intake, illicit drugs, and certain medications [6].

\section{Impact of Parafunctional Behaviors on Dental Health}

Bruxism and other parafunctional behaviors were thought to have a major negative impact on dental health. They were reported to result in wearing down of enamel or teeth wear, teeth fracture, wear, or loss that necessitate treatment with bridges, crowns, or implants. Furthermore, they were thought to worsen the general condition of stomatognathic system. In advanced untreated cases, temporomandibular joints can be affected [2]. However, more recent researches indicate that other factors such as teeth erosion are involved [17]. Furthermore, discrepancy of results between studies is mainly attributed to the definition and diagnosis of bruxism. Studies that had included patients who self-reported bruxism showed a significant correlation between bruxism and teeth wear $[18,19]$, whereas studies which included polysomnographic-diagnosed patients reported that there was no considerable relationship between bruxism and tooth wear or temporomandibular joint dysfunction [20].

\section{Parafunctional Behaviors and Dental Bridges}

Not only do the parafunctional behaviors affect the teeth, but they also have deleterious effects on dental protheses including dental bridges. Parafunctional behaviors, particularly bruxism, can dislodge or damage dental bridges. The deleterious damage on dental bridges caused by persistent bruxism constitutes a major financial burden. The average cost of three-unit bridge is $\$ 3,800$, and the average cost of four-unit bridge is $\$ 7,000$. Tomonaga et al [21] in their study about the correlation between sleep bruxism and dislodgement of dental restoration found that among the severity of sleep bruxism was a significant impact on shortening the duration to dislodgement of dental restorations (including bridges, crowns, jointed crowns, or inlay). Many researchers reported that bruxism was significantly associated with failure of fixed dental protheses on the long-term follow-up [22, 23]. Bruxism and parafunctional behaviors resulted in early damage and shortened survival of dental prostheses [24]. Gold and ceramic materials are more preferred than resins by clinicians in treating patients with bruxism because they are more resistant to damage and sub- 
stance loss [25].

Along with the impact of parafunctional behaviors on fixed dental protheses, researchers stated that such behaviors, including bruxism, had shortened the longevity of removable partial dentures [26]. As regards complete dentures, studies have depicted that bruxism had negative consequences on the residual dentition and denture-bearing tissues among those patients [27]. Furthermore, using a protective night device for the fixed prothesis had improved the bruxism related complications among the studied patients [28].

\section{Conclusions}

Parafunctional behaviors are common involuntary movements involving the masticatory system. They are not uncommon among the population, especially children. These behaviors have deleterious effects on dental structures. Causes of parafunctional behaviors include anxiety, depression, smoking, caffeine intake, sleep disorders, or central neurotransmitter dysfunction. Bruxism and other similar masticatory system activity cause dental fracture, loss, and weardown of enamel or teeth. They can also affect different types of dental protheses both fixed and removable types. Parafunctional behaviors shorten the life expectancy of these protheses, and damage residual dentition and denture-bearing tissues.

\section{Acknowledgments}

The authors would like to thank all the contributors in this article, and to the Ministry of Health for providing us with the access to many databases for our research.

\section{Disclosures}

The authors declare that no competing or financial interests exist.

\section{References}

1. Mills CS. International Association of Orofacial Myology History: origin - background - contributors. Int J Orofacial Myology. 2011;37:5-25.

2. Fragoso YD, Alves HH, Garcia SO, Finkelsztejn A. Prevalence of parafunctional habits and temporomandibular dysfunction symptoms in patients attending a tertiary headache clinic. Arq Neuropsiquiatr. 2010;68(3):377380.

3. Melchior M de O, Mazzetto MO, de Felicio CM. Temporomandibular disorders and parafunctional oral habits: an anamnestic study. Dental Press J Orthod. 2012;17(2):8389.

4. Heimer MV, Tornisiello Katz CR, Rosenblatt A. Nonnutritive sucking habits, dental malocclusions, and facial morphology in Brazilian children: a longitudinal study.
Eur J Orthod. 2008;30(6):580-585.

5. Feteih RM. Signs and symptoms of temporomandibular disorders and oral parafunctions in urban Saudi Arabian adolescents: a research report. Head Face Med. 2006;2:25.

6. Shetty S, Pitti V, Satish Babu CL, Surendra Kumar GP, Deepthi BC. Bruxism: a literature review. J Indian Prosthodont Soc. 2010;10(3):141-148.

7. Bender SD. Occlusion, function, and parafunction: understanding the dynamics of a healthy stomatagnathic system educational objectives. 2009.

8. Manfredini D, Lobbezoo F. Role of psychosocial factors in the etiology of bruxism. J Orofac Pain. 2009;23(2):153166.

9. Murali RV, Rangarajan P, Mounissamy A. Bruxism: Conceptual discussion and review. J Pharm Bioallied Sci. 2015;7(Suppl 1):S265-270.

10. Lobbezoo F, Van Der Zaag J, Naeije M. Bruxism: its multiple causes and its effects on dental implants - an updated review. J Oral Rehabil. 2006;33(4):293-300.

11. Bertrand PM, Black DF, Carlson CR. American academy of orofacial pain guidelines for assessment, diagnosis, and management. Pain. 2008;316.

12. Reddy SV, Kumar MP, Sravanthi D, Mohsin AH, Anuhya V. Bruxism: a literature review. J Int Oral Health. 2014;6(6):105-109.

13. Thorpy MJ. Classification of sleep disorders. Neurotherapeutics. 2012;9(4):687-701.

14. The glossary of prosthodontic terms. J Prosthet Dent. 2005;94(1):10-92.

15. De Laat A, Macaluso GM. Sleep bruxism as a motor disorder. Mov Disord. 2002;17(Suppl 2):S67-69.

16. Suneel VB, Kotian S, Jujare RH, Shetty AK, Nidhi S, Grover S. Incidence of Prosthetic Complications associated with Implant-borne Prosthesis in a Sleep Disorder Center. J Contemp Dent Pract. 2017;18(9):821-825.

17. Johansson A, Johansson AK, Omar R, Carlsson GE. Rehabilitation of the worn dentition. J Oral Rehabil. 2008;35(7):548-566.

18. Johansson A, Unell L, Carlsson GE, Soderfeldt B, Halling A. Differences in four reported symptoms related to temporomandibular disorders in a cohort of 50-year-old subjects followed up after 10 years. Acta Odontol Scand. 2008;66(1):50-57.

19. Johansson A, Omar R, Carlsson GE. Bruxism and prosthetic treatment: a critical review. J Prosthodont Res. 2011;55(3):127-136.

20. Baba K, Haketa T, Clark GT, Ohyama T. Does tooth wear status predict ongoing sleep bruxism in 30-year-old Japanese subjects? Int J Prosthodont. 2004;17(1):39-44.

21. Tomonaga A, Ikeda M, Kato H, Ohata N. [Influence of sleep bruxism on dislodgement of dental restorations]. Nihon Hotetsu Shika Gakkai Zasshi. 2005;49(2):221230.

22. Pjetursson BE, Bragger U, Lang NP, Zwahlen M. Comparison of survival and complication rates of tooth-supported fixed dental prostheses (FDPs) and implant-supported FDPs and single crowns (SCs). Clin Oral Implants Res. 2007;18(Suppl 3):97-113.

23. Pjetursson BE, Lang NP. Prosthetic treatment plan- 
ning on the basis of scientific evidence. J Oral Rehabil. 2008;35(Suppl 1):72-79.

24. Bragger U, Aeschlimann S, Burgin W, Hammerle CH, Lang NP. Biological and technical complications and failures with fixed partial dentures (FPD) on implants and teeth after four to five years of function. Clin Oral Implants Res. 2001;12(1):26-34.

25. Yip KH, Smales RJ, Kaidonis JA. Differential wear of teeth and restorative materials: clinical implications. Int J Prosthodont. 2004;17(3):350-356.
26. Creugers NH, Witter DJ, Van't Spijker A, Gerritsen AE, Kreulen CM. Occlusion and temporomandibular function among subjects with mandibular distal extension removable partial dentures. Int J Dent. 2010;2010:807850.

27. CGC Zarb GA, Bolender CL, Hickey JC. Boucher's prosthodontic treatment for edentulous patients. 10th edn. St. Louis C.V.Mosby. 1990;1-27.

28. Celebic A, Knezovic-Zlataric D. A comparison of patient's satisfaction between complete and partial removable denture wearers. J Dent. 2003;31(7):445-451. 